\title{
Incidence de la mortalité par intoxication sur l'espérance de vie à la naissance au Canada (2000-2016)
}

\author{
Heather M. Orpana, Ph. D. (1,2); Justin J. Lang, Ph. D. (1); Diana George, M. Sc. (1); Jessica Halverson, M.T.S., M.S.P. (1)
}

\section{Résumé}

L'augmentation de la mortalité liée aux opiö̈des a contribué à des baisses de l'espérance de vie à la naissance aux États-Unis et en Colombie-Britannique. Nous avons analysé les données nationales sur la mortalité au Canada entre 2000 et 2016 afin de déterminer dans quelle mesure les décès liés aux intoxications avaient influencé l'espérance de vie à la naissance selon le groupe d'âge et le sexe. Entre 2000 et 2016, l'espérance de vie à la naissance a augmenté de presque trois ans, mais la mortalité attribuable aux intoxications accidentelles, dont celles par opioïdes, a réduit cette hausse de 0,16 an. Même si l'espérance de vie à la naissance n'a pas globalement diminué au Canada pendant cette période, les tendances actuelles laissent présager que la crise nationale des surdoses d'opioïdes va continuer à amoindrir les gains relatifs à l'espérance de vie.

\section{Introduction}

Le Canada et les États-Unis ont récemment enregistré des hausses saisissantes de décès liés aux opioïdes. On a déjà comptabilisé plus de 9000 décès apparemment liés aux opioïdes au Canada entre janvier 2016 et juin 2018. Aux États-Unis, en 2016, les opioïdes étaient en cause dans environ 42000 décès par surdose, soit une hausse de 21,5\% du taux de mortalité par surdose (intoxication) ajusté selon l'âge en comparaison de $2015^{2}$. Ce fardeau croissant de la mortalité, qui touche de façon disproportionnée les jeunes adultes ${ }^{1,2}$, pourrait influencer l'espérance de vie dans les zones concernées. Bien que l'espérance de vie à la naissance ait augmenté de deux ans entre 2000 et 2015 aux États-Unis, la mortalité attribuable à une intoxication par des drogues ou des médicaments a plus que doublé pendant la même période. Ces décès représentent une perte de 0,28 an d'espérance de vie à la naissance, et les intoxications aux opioïdes comptent pour 0,21 an dans celle-ci ${ }^{3}$. Simplement entre 2015 et 2016, Kochanek et ses collaborateurs ont rapporté une baisse globale de 0,1 an de l'espérance de vie à la naissance aux ÉtatsUnis, en raison d'une diminution de 0,2 an chez les hommes sans variation de l'espérance de vie des femmes ${ }^{4}$. La ColombieBritannique - l'une des provinces canadiennes où la crise des surdoses d'opioïdes s'est manifestée en premier et a engendré les plus lourdes conséquences ${ }^{5}$ - a signalé une baisse de 0,38 an de l'espérance de vie à la naissance entre 2014 et 2016, le tiers de cette baisse étant attribuée aux surdoses de drogues illicites, principalement les opioİdes $^{6}$. L'incidence de la crise des surdoses d'opioïdes sur l'espérance de vie à la naissance à l'échelle du Canada n'a pas encore été établie.

Notre étude visait à étudier l'évolution de l'espérance de vie à la naissance au Canada entre 2000 et 2016, et plus spécifiquement entre 2014 et 2016, en ciblant les intoxications accidentelles, délibérées et d'intention indéterminée. Les changements relatifs à l'espérance de vie ont été décomposés selon la cause de décès, le groupe d'âge et le sexe. Ces analyses vont favoriser une meilleure compréhension de la manière dont la mortalité par intoxication aux opioïdes influe sur l'espérance de vie à la naissance au Canada.

\section{Méthodologie}

Les données des statistiques de l'état civil proviennent du tableau 13-10-0156-01 de la Base de données sur les décès ${ }^{7}$ et les estimations de population proviennent du tableau 17-10-0005-01 de Statistique Canada ${ }^{8}$. Les données sur la mortalité ont été réparties dans les catégories suivantes : intoxication accidentelle par des narcotiques et exposition à ces produits [X42], autointoxication par des narcotiques et exposition à ces produits [X62], intoxication par des narcotiques et exposition à ces produits, intention non déterminée [Y12], autres intoxications accidentelles [X40, X41, X43-49], autres auto-intoxications délibérées [X60, X61, X63-X69], autres intoxications d'intention indéterminée [Y10, Y11, Y13-Y19], autres lésions auto-infligées [X70X84], accidents liés au transport [V01-V99], autres causes externes que celles déjà incluses [W00-W99, X00-X39, X50-X59, Y20Y89] et causes résiduelles (tous les autres chapitres de la CIM-10).

Des tables de mortalité abrégées ont été produites en fonction des groupes d'âge normalisés : moins d'un an, de 1 à 4 ans, puis par tranche de cinq ans jusqu'au

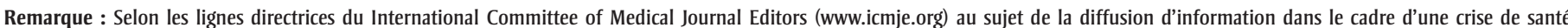

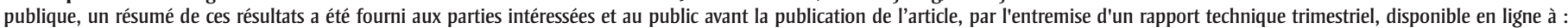

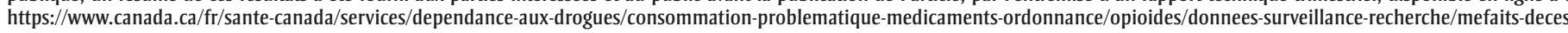
/mesurer-impact-sur-esperance-vie.html

Rattachement des auteurs :

1. Agence de la santé publique du Canada, Ottawa (Ontario), Canada

2. École d'épidémiologie et de santé publique, Université d'Ottawa, Ottawa (Ontario), Canada

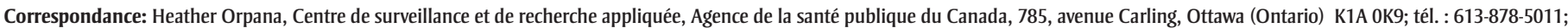
courriel : heather.orpana@canada.ca 
dernier groupe, celui des 90 ans et plus. L'espérance de vie à la naissance a été calculée selon la méthode de Chiang', et la méthode décrite par Hsieh ${ }^{10}$ a servi à l'estimation de la dernière valeur contenue dans la table de mortalité. L'espérance de vie du moment correspond au nombre d'années qu'un individu va vivre s'il suit les probabilités de décès par âge et par sexe observées au cours d'une période donnée. Nous avons suivi la méthode d'Arriaga pour décomposer les variations de l'espérance de vie selon le groupe d'âge, le sexe et la cause de décès, au moyen de feuilles de calcul Excel créées par Auger et collab. ${ }^{11}$. Cette approche tient compte à la fois des effets directs et des effets indirects du groupe d'âge, du sexe et de la cause de décès. Les analyses ont été menées globalement pour les périodes 2000-2016 et 2014-2016 ainsi que pour les hommes et les femmes séparément pour 2000-2016. Nous avons également examiné dans quelle mesure chaque groupe d'âge et chaque cause contribuaient à la différence entre l'espérance de vie à la naissance des hommes et celle des femmes en 2016.

\section{Résultats}

L'espérance de vie à la naissance a augmenté de presque trois ans entre 2000 et 2016, passant de 79,27 à 82,25 ans pour les hommes et les femmes réunis. La majorité des hausses observées sont attribuables aux causes résiduelles autres que les blessures (tableau 1). Cependant, alors que la catégorie des décès par blessure s'est révélée être globalement à l'origine d'une faible augmentation de l'espérance de vie à la naissance $(0,11 \mathrm{an})$, deux causes de décès comprises dans cette catégorie ont influencé à la baisse l'espérance de vie à la naissance des hommes et des femmes combinés : les intoxications accidentelles par des narcotiques et l'exposition à ces produits $(-0,08)$ et les autres intoxications accidentelles $(-0,08)$. La hausse de l'espérance de vie à la naissance entre 2000 et 2016 a été plus marquée chez les hommes (3,48 ans) que chez les femmes (2,52 ans), et parallèlement l'effet négatif des intoxications accidentelles (tant par narcotiques que par d'autres substances) sur l'espérance de vie s'est révélé plus prononcé chez les hommes
$(-0,23$ an) que chez les femmes $(-0,09 \mathrm{an})$. Mis à part les " autres causes de blessure » pour les femmes entre 2000 et 2016, toutes les catégories de décès par blessure ont eu un effet nul sur l'espérance de vie ou ont contribué à sa hausse. De toutes les causes associées aux blessures, c'est la diminution des décès attribuables aux accidents liés au transport qui a le plus contribué à l'augmentation de l'espérance de vie. L'incidence des intoxications accidentelles (par des narcotiques ou par d'autres substances) sur l'espérance de vie à la naissance est manifeste principalement depuis 2014. Entre 2014 et 2016, l'espérance de vie à la naissance a globalement augmenté de 0,53 an pour les hommes et les femmes combinés (tableau 1), mais les intoxications accidentelles ont entraîné une perte de 0,09 an.

En 2016, l'espérance de vie à la naissance des hommes était inférieure de 4,20 ans à celle des femmes (tableau 1). La mortalité supérieure associée aux intoxications accidentelles par des narcotiques et l'exposition à ces produits chez les hommes

TABLEAU 1

Répartition des contributions par cause aux différences relatives à l'espérance de vie à la naissance entre 2000, 2014 et 2016 (ensemble, hommes et femmes) et entre les hommes et les femmes en 2016

\begin{tabular}{|c|c|c|c|c|c|c|c|}
\hline & \multirow{2}{*}{ Catégorie } & \multirow{2}{*}{ s de cause de décès } & \multicolumn{3}{|c|}{2000 à 2016} & \multirow{2}{*}{$\begin{array}{c}\begin{array}{c}2014 \text { à } \\
2016\end{array} \\
\text { Ensemble }\end{array}$} & \multirow{2}{*}{ 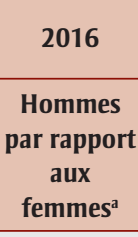 } \\
\hline & & & \multirow{2}{*}{$\begin{array}{c}\text { Ensemble } \\
-0,08\end{array}$} & \multirow{2}{*}{$\begin{array}{c}\text { Hommes } \\
-0,12\end{array}$} & \multirow{2}{*}{$\begin{array}{c}\text { Femmes } \\
-0,04\end{array}$} & & \\
\hline $\begin{array}{l}\text { Causes de décès } \\
\text { associées à des } \\
\text { blessures }\end{array}$ & $\begin{array}{l}\text { Intoxication } \\
\text { accidentelle }\end{array}$ & $\begin{array}{l}\text { Intoxication accidentelle par des narcotiques et } \\
\text { psychodysleptiques [hallucinogènes], et exposition à } \\
\text { ces produits, non classés ailleurs [X42] }\end{array}$ & & & & \multirow{2}{*}{$\begin{array}{l}-0,04 \\
-0,05\end{array}$} & \multirow{2}{*}{$\begin{array}{l}-0,13 \\
-0,09\end{array}$} \\
\hline & & $\begin{array}{l}\text { Autres intoxications accidentelles } \\
\text { [X40, X41, X43 à X49] }\end{array}$ & $-0,08$ & $-0,11$ & $-0,05$ & & \\
\hline & \multirow[t]{2}{*}{ Intoxication délibérée } & $\begin{array}{l}\text { Auto-intoxication par des narcotiques et } \\
\text { psychodysleptiques [hallucinogènes] et exposition à } \\
\text { ces produits, non classés ailleurs [X62] }\end{array}$ & 0,00 & 0,00 & 0,00 & 0,00 & 0,00 \\
\hline & & Autres intoxications délibérées [X60, X61, X63 à X69] & 0,02 & 0,04 & 0,00 & 0,01 & $-0,01$ \\
\hline & $\begin{array}{l}\text { Intoxication d'intention } \\
\text { indéterminée }\end{array}$ & $\begin{array}{l}\text { Autres intoxications d'intention indéterminée } \\
\text { [Y10, Y11, Y13 à Y19] }\end{array}$ & 0,01 & 0,01 & 0,00 & 0,03 & 0,00 \\
\hline & \multicolumn{2}{|c|}{ Autres lésions auto-infligées [X70 à X84] } & 0,02 & 0,05 & 0,03 & 0,01 & $-0,33$ \\
\hline & \multicolumn{2}{|c|}{ Accidents liés au transport [V01 à V99] } & 0,16 & 0,22 & 0,10 & 0,02 & $-0,14$ \\
\hline & \multicolumn{2}{|c|}{ Autres blessures [W00-W99, X00-X39, X50-X59, Y20-Y89] } & 0,06 & 0,10 & $-0,02$ & 0,04 & $-0,14$ \\
\hline \multicolumn{3}{|c|}{ Causes résiduelles de décès [tous les autres chapitres de la CIM-10] } & 2,88 & 3,30 & 2,50 & 0,49 & $-3,35$ \\
\hline \multicolumn{3}{|c|}{ Variation totale de l'espérance de vie } & 2,99 & 3,48 & 2,52 & 0,53 & $-4,20$ \\
\hline
\end{tabular}

Remarque : Les estimations ayant été arrondies, il se peut que leur somme ne corresponde pas au total de la colonne.

${ }^{a}$ Les valeurs négatives représentent une contribution négative à la différence de l'espérance de vie des hommes par rapport à celle des femmes. 
compte pour 0,13 an dans cet écart, alors que la contribution des autres intoxications accidentelles atteint 0,09 an. Parmi toutes les autres causes de décès par blessure, ce sont les autres lésions auto-infligées qui contribuaient le plus fortement $(0,33$ an) à l'écart entre les sexes.

Comme l'illustre le tableau 2, la plupart des groupes d'âge ont concouru à la hausse de l'espérance de vie à la naissance entre 2000 et 2016, chez les hommes comme chez les femmes. Néanmoins, le groupe des 25 à 29 ans et celui des 30 à 34 ans chez les hommes ont eu un léger effet à la baisse. Entre 2014 et 2016, pour les deux sexes combinés, presque tous les groupes d'âge, de celui des 10 à 14 ans à celui des 45 à 49 ans, ont conduit à une légère baisse de l'espérance de vie à la naissance, mais cet effet a été compensé par les gains attribuables aux adultes de 55 à 59 ans et les plus âgés.
La figure 1 montre la contribution de chaque cause à l'évolution entre 2000 et 2016 de l'espérance de vie à la naissance, selon le groupe d'âge et pour les deux sexes combinés. La plupart des gains relatifs à l'espérance de vie concernent les adultes plus âgés et les causes résiduelles autres que les blessures. Toutefois, les intoxications accidentelles par des narcotiques et l'exposition à ces produits, de même que les autres intoxications accidentelles, ont retranché au moins 0,01 an à l'espérance de vie dans les groupes d'âge compris entre 20 et 59 ans.

\section{Analyse}

L'espérance de vie à la naissance continue d'augmenter à l'échelle nationale au Canada. Néanmoins, les gains réalisés entre 2000 et 2016 et plus spécifiquement entre 2014 et 2016 ont été modérés par l'augmentation des décès attribuables aux intoxications

TABLEAU 2

Répartition de la contribution par âge à l'évolution de l'espérance de vie à la naissance entre 2000, 2014 et 2016 (ensemble, hommes et femmes) et entre les hommes et les femmes, 2016

\begin{tabular}{|c|c|c|c|c|c|}
\hline \multirow[b]{2}{*}{$\begin{array}{l}\text { Groupe d'âge } \\
\text { (ans) }\end{array}$} & \multicolumn{3}{|c|}{2000 à 2016} & \multirow{2}{*}{$\frac{2014 \text { à } 2016}{\text { Ensemble }}$} & \multirow{2}{*}{$\begin{array}{c}2016 \\
\text { Hommes par } \\
\text { rapport aux } \\
\text { femmes }^{\mathrm{a}}\end{array}$} \\
\hline & Ensemble & Hommes & Femmes & & \\
\hline Moins de 1 & 0,05 & 0,09 & 0,01 & 0,02 & $-0,02$ \\
\hline 1 à 4 & 0,01 & 0,01 & 0,01 & 0,00 & $-0,01$ \\
\hline 5 à 9 & 0,02 & 0,02 & 0,01 & 0,00 & 0,00 \\
\hline 10 à 14 & 0,01 & 0,01 & 0,01 & $-0,01$ & $-0,01$ \\
\hline 15 à 19 & 0,05 & 0,07 & 0,03 & 0,00 & $-0,06$ \\
\hline 20 à 24 & 0,03 & 0,05 & 0,02 & $-0,02$ & $-0,12$ \\
\hline 25 à 29 & $-0,01$ & $-0,02$ & 0,00 & $-0,01$ & $-0,15$ \\
\hline 30 à 34 & 0,00 & $-0,01$ & 0,00 & $-0,01$ & $-0,13$ \\
\hline 35 à 39 & 0,03 & 0,04 & 0,01 & 0,00 & $-0,10$ \\
\hline 40 à 44 & 0,05 & 0,07 & 0,04 & $-0,01$ & $-0,11$ \\
\hline 45 à 49 & 0,06 & 0,08 & 0,04 & $-0,01$ & $-0,13$ \\
\hline 50 à 54 & 0,10 & 0,12 & 0,08 & 0,00 & $-0,16$ \\
\hline 55 à 59 & 0,16 & 0,21 & 0,11 & 0,03 & $-0,24$ \\
\hline 60 à 64 & 0,24 & 0,32 & 0,16 & 0,03 & $-0,33$ \\
\hline 65 à 69 & 0,35 & 0,45 & 0,23 & 0,04 & $-0,40$ \\
\hline 70 à 74 & 0,44 & 0,57 & 0,31 & 0,09 & $-0,42$ \\
\hline 75 à 79 & 0,46 & 0,57 & 0,37 & 0,07 & $-0,47$ \\
\hline 80 à 84 & 0,40 & 0,45 & 0,38 & 0,08 & $-0,49$ \\
\hline 85 à 89 & 0,30 & 0,26 & 0,38 & 0,09 & $-0,43$ \\
\hline 90 et plus & 0,22 & 0,13 & 0,32 & 0,16 & $-0,42$ \\
\hline Total & 2,99 & 3,48 & 2,52 & 0,53 & $-4,20$ \\
\hline
\end{tabular}

Remarque : Les estimations ayant été arrondies, il se peut que leur somme ne corresponde pas au total de la colonne.

${ }^{a}$ Les valeurs négatives représentent une contribution négative à la différence de l'espérance de vie des hommes par rapport à celle des femmes. accidentelles, tant par des narcotiques que par d'autres substances. S'il n'y a pas eu de déclin global de l'espérance de vie à la naissance sur le plan national, comme ce fut le cas aux États-Unis ou à l'échelle provinciale en Colombie-Britannique ${ }^{3-5}$, la mortalité varie certainement fortement d'une région à l'autre du Canada. Il se pourrait également que l'épidémie de surdoses d'opioïdes ait touché relativement plus tôt la Colombie-Britannique et les États-Unis que les autres régions du Canada. L'absence de déclin global ne signifie donc pas pour autant qu'il n'y ait pas de crise de santé publique au Canada, car une réduction de l'espérance de vie constitue toujours une situation exceptionnelle. D'après les données sur l'espérance de vie à la naissance compilées par Statistique Canada entre 1921 et 2011, les seules baisses visibles d'une année à l'autre ont été enregistrées pendant quelques périodes au cours des années 1920 et $1930^{12}$. Il convient de noter que les intoxications accidentelles par des narcotiques et l'exposition à ces produits d'une part et les autres intoxications accidentelles d'autre part ont eu une contribution négative comparable en matière d'espérance de vie à la naissance. Dans cette étude, nous avons utilisé les codes correspondant aux causes de décès de la CIM-10 mais non les codes $\mathrm{T}$ de diagnostic associés, en raison de restrictions concernant la divulgation des données. Par conséquent, il est probable que les opioïdes n'étaient pas les seuls responsables des décès liés aux intoxications accidentelles par des narcotiques et à l'exposition à ces produits [X42]. À l'inverse, certaines autres intoxications accidentelles [X40, X41, X4349] pourraient être associées aux opioïdes. Comme l'augmentation des décès a suivi des tendances similaires dans les deux catégories, il est important de tenir compte de la catégorie des intoxications accidentelles dans son ensemble. Aux États-Unis, les opioïdes ont été associés à une perte de 0,21 an sur la perte totale de 0,28 an attribuable aux intoxications accidentelles ${ }^{3}$, et la Colombie-Britannique a signalé que les surdoses de drogues illicites avaient fait baisser de 0,12 an l'espérance de vie à la naissance, sur un déclin total de 0,38 an entre 2014 et 20166. Les données nationales disponibles révèlent qu'au moins un autre type de substance était aussi en cause dans environ $76 \%$ des décès accidentels apparemment liés aux opioïdes qui sont survenus au Canada entre janvier 2016 et juin $2018^{1}$. Notre analyse gagnerait en précision si les codes $\mathrm{T}$ de diagnostic étaient intégrés. Au moment de notre analyse, les 
FIGURE 1

Contributions par cause à l'évolution de l'espérance de vie à la naissance selon le groupe d'âge, hommes et femmes combinés, $2000-2016$

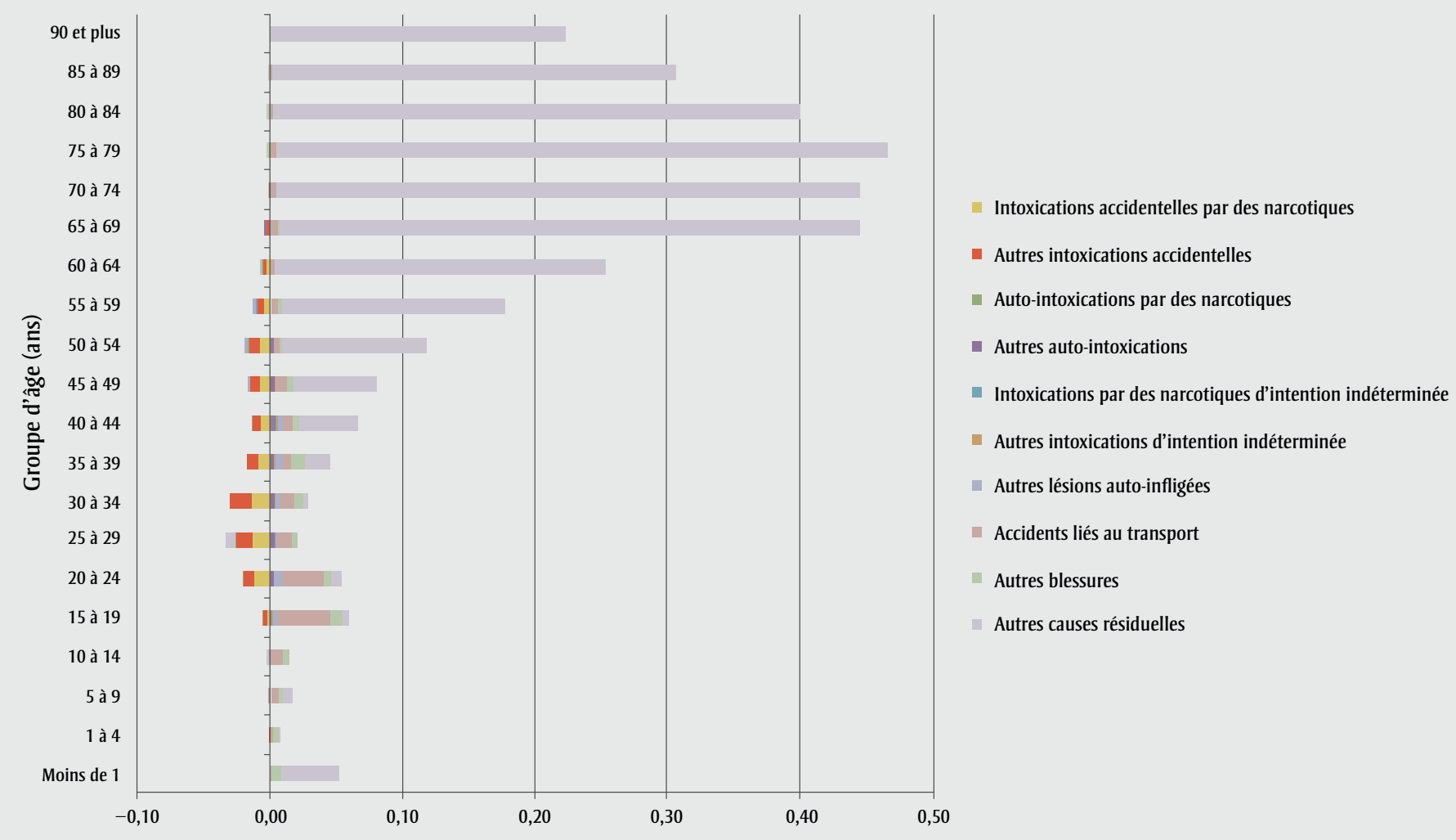

Évolution de l'espérance de vie entre 2000 et 2016 (ans)

données complètes sur les décès s'arrêtaient en 2016. Une mise à jour sera effectuée à la publication des données finales sur les décès pour 2017. Étant donnée l'augmentation notable des décès apparemment liés aux opioïdes en 2017 par rapport à 2016, et à la lumière des données préliminaires sur les décès pour la première moitié de 2018, tout porte à croire que l'incidence sur l'espérance de vie va continuer à s'accentuer ${ }^{1}$. Comme nous n'avons pas mené d'analyses à l'échelle régionale, les résultats que nous présentons ici brossent un tableau valable uniquement à l'échelle nationale. Les prochaines analyses vont pouvoir porter sur les variations régionales potentielles en ce qui concerne l'effet des décès liés aux intoxications sur l'évolution de l'espérance de vie.

Il ressort en outre que les décès attribuables à l'auto-intoxication par des narcotiques et l'exposition à ces produits, de même que les décès attribuables à l'intoxication d'intention indéterminée par des narcotiques et l'exposition à ces produits, n’ont pas affaibli l'augmentation de l'espérance de vie à l'échelle nationale pendant les périodes à l'étude. La crise des surdoses d'opioïdes n'aurait donc pas influencé les taux de mortalité liés à l'auto-intoxication par des narcotiques et l'exposition à ces produits à l'échelle du pays, comme le montrent des données récentes de l'Alberta ${ }^{13}$.

Bien que la diminution associée à la plupart des causes de décès par blessure ait favorisé des gains d'espérance de vie à la naissance au Canada, les intoxications accidentelles ont ralenti la progression globale de l'espérance de vie à la naissance pendant les périodes à l'étude, chez les hommes comme chez les femmes. Les intoxications accidentelles par narcotiques et l'exposition à ces produits, de même que les autres intoxications accidentelles, ont contribué à ce ralentissement. Même si l'espérance de vie à la naissance continue d'augmenter au Canada et suit des tendances similaires à celles observées dans les pays comparables à revenu élevé ${ }^{14,15}$, la crise actuelle des surdoses d'opiö̈des pourrait continuer à affaiblir cette hausse, vu la fréquence accrue des décès attribuables aux intoxications accidentelles. Les résultats pourraient évoluer avec la publication des nouvelles données, compte tenu de l'augmentation relative des décès liés aux opioïdes ayant été signalés en 2017 et des données concernant la première moitié de $2018^{1}$.

\section{Remerciements}

Nous tenons à souligner la contribution de N. Auger de l'INSPQ et de ses collaborateurs, qui ont développé le programme Excel que nous avons utilisé pour analyser les différences relatives à l'espérance de vie.

\section{Conflits d'intérêts}

Nous déclarons n'avoir aucun conflit d'intérêts relativement à ces travaux.

\section{Contributions des auteurs et avis}

JH et DG ont élaboré le projet. $\mathrm{HO}$ a procédé à l'analyse des données et a rédigé 
l'article. JJL, DG et JH ont participé à l'interprétation des données et ont effectué une analyse critique de l'article.

Le contenu de l'article et les points de vue qui y sont exprimés n'engagent que les auteurs; ils ne correspondent pas nécessairement à ceux du gouvernement du Canada.

\section{Références}

1. Comité consultatif spécial sur l'épidémie de surdoses d'opioïdes. Rapport national : Décès apparemment liés à la consommation d'opioïdes au Canada (janvier 2016 à juin 2018) [Internet]. Ottawa (Ont.) : Agence de la santé publique du Canada; 2018 [consultation le 17 décembre 2018]. En ligne à : https://infobase.phac-aspc.gc.ca /datalab/surveillance-nationale-opioides -mortalite.html

2. National Centre for Injury Prevention. Drug overdose death data [Internet]. Atlanta (GA): Centres for Disease Control and Prevention; 2017 [consultation le 12 novembre 2018]. En ligne à : https://www.cdc.gov/drugoverdose /data/statedeaths.html

3. Dowell D, Arias E, Kochanek K, et al. Contribution of opioid-involved poisoning to the change in life expectancy in the United States, 2000-2015. JAMA. 2017;318(11):1065-1067. doi: 10.1001 /jama.2017.9308.

4. Kochanek KD, Murphy SL, Xu JQ, et al. Mortality in the United States, 2016. NCHS Data Brief, no 293. Hyattsville (MD): National Center for Health Statistics; 2017. En ligne à : https:// www.cdc.gov/nchs/data/databriefs /db293.pdf

5. Government of British Columbia. Provincial health officer declares public health emergency [Internet]. Victoria (BC): Government of British Columbia; 2016. En ligne à : https:// news.gov.bc.ca/releases/2016HLTH0026 $-000568$

6. Ye X, Sutherland J, Henry B, et al. Aperçu - Impact des décès par surdose de drogue sur l'espérance de vie à la naissance en Colombie-Britannique. Promotion de la santé et prévention des maladies chroniques au Canada, 2018;38(6):282-286. doi : 10.24095 /hpcdp.38.6.05f.
7. Statistique Canada. Tableau 13-100156-01 : Décès, selon la cause, Chapitre XX : Causes externes de morbidité et de mortalité (V01 à Y89) [Internet]. Ottawa (Ont.) : Statistique Canada; 2018. En ligne à : https:// www150.statcan.gc.ca/t1/tbl1/fr/tv .action?pid $=1310015601$

8. Statistique Canada. Tableau 17-100005-01: Estimations de la population au $1^{\text {er }}$ juillet, par âge et sexe. Ottawa (Ont.) : Statistique Canada; 2018. En ligne à : https://www150.statcan .gc.ca/t1/tbl1/fr/tv.action?pid $=1710000501$

9. Chiang CL. The life table and its applications. Malabar (FL): Robert E. Krieger Publishing Company; 1984.

10. Hsieh JJ. A general theory of life table construction and a precise abridged life table method. Biom J. 1991;33(2): 143-162.

11. Auger N, Feuillet P, Martel S, et al. Mortality inequality in populations with equal life expectancy: a practical decomposition method in SAS and Excel. Ann Epidemiol. 2014;24(8):575580. doi: 10.1016/j.annepidem.2014 .05 .006 .

12. Decady Y, Greenberg L. Quatre-vingtdix ans de changements dans l'espérance de vie [Internet]. Ottawa (Ont.) : Statistique Canada; 2014. En ligne à : https://www150.statcan.gc.ca/n1/pub /82-624-x/2014001/article/14009-fra.htm

13. Chan EYL, McDonald BM, Brooks-Lim EJG, et al. Aperçu - Suicides et intoxication aux opioïdes en Alberta (20002016). Promotion de la santé et prévention des maladies chroniques au Canada. 2018;38(9):388-392. doi : 10.24095/hpcdp.38.9.07.

14. GBD 2016 Mortality Collaborators. Global, regional, and national under-5 mortality, adult mortality, age-specific mortality, and life expectancy, 19702016: a systematic analysis for the Global Burden of Disease Study 2016. The Lancet. 2017;390(10100):1084-1150.

15. Lang JJ, Alam S, Cahill LE, et al. Global Burden of Disease Study trends for Canada from 1990 to 2016. CMAJ. 2018;190(44):E1296-E1304. doi: 10.1503 /cmaj.180698. 